\title{
Application of Auriculotherapy for Post-Burn Scar Syndrome in Young Adults with Major Burns
}

\author{
Cha-Chun Chen ${ }^{1,2,3}$, San-Pei Chen ${ }^{4}$, Shu-Yu Lyu ${ }^{3}$, Chung-Hua Hsu ${ }^{2,5, *}$ \\ ${ }^{1}$ Department of Plastic Surgery, Shin Kong Wu Ho-Su Memorial Hospital, Taipei, Taiwan \\ 2Institute of Traditional Medicine, School of Medicine, National Yang-Ming University, Taipei, Taiwan \\ ${ }^{3}$ Department of Leisure Industry and Health Promotion, National Taipei University of Nursing Health Sciences, Taipei, Taiwan \\ ${ }^{4}$ Department of Sport and Health Management, Da-Yeh University, Changhua, Taiwan \\ ${ }^{5}$ Taipei City Hospital, Taipei, Taiwan
}

Received October 15, 2019

Revised June 5, 2021

Accepted July 2, 2021

Correspondence to Chung-Hua Hsu

Institute of Traditional Medicine, School of Medicine, National Yanh-Ming University, Taipei, Taiwan

E-mail owlherbs@yahoo.com.tw; jillclocl@gmail.com
Background: A burn scar is a type of hypertrophic scar that can cause significant clinical symptoms, discomfort, and post-burn scar (PBS) syndrome in up to $77 \%$ of patients with burn injuries. Medication and rehabilitation are rarely effective at managing patient discomfort, and both laser and surgical interventions are postponed until the scar stabilizes and discomfort is tolerable.

Objectives: The present study was conducted to investigate the effectiveness of auricular acupuncture among burn victims from the Formosa Color Dust Explosion in Taiwan.

Methods: We enrolled 31 victims of the 2016 Formosa Color Dust Explosion who met the study inclusion criteria. The intervention involved placement of magnetic beads over the auricular Shenmen and Subcortex acupoints on one ear. Patients performed selfmassage five times per day, and both magnet beads were removed between the fifth and seventh days during the sessions. Several evaluation tools were used to assess clinical symptoms: the visual analogue scale for pain assessment, Burn Man Itch Scale for perceived patient itchiness, 5-D Pruritus Scale for sleep quality, and heart rate variability (HRV) for effects on the autonomic nervous system.

Results: The clinical symptoms were significantly decreased following the intervention, but the effect did not endure. The normal-to-normal heart rate interval, heart rate analysis abnormalities, and very low frequency heart rate were significantly decreased among patients with abnormal HRV (SD < 40) following treatment.

Conclusion: Stimulation of the auricular Shenmen and Subcortex acupoints may effectively reduce pain, itchiness, and sleep disturbances among patients with PBS syndrome.

Keywords: Post-burn scar syndrome, Auriculotherapy, Case study, Burn therapy

\section{INTRODUCTION}

A burn scar is a type of hypertrophic scar that can engender significant clinical symptoms, discomfort, and post-burn scar (PBS) syndrome [1,2]. Up to $77 \%$ of patients with burn injuries develop PBS syndrome and are consequently more likely to present with pain and pruritus (itching) [1]. These symptoms are caused by hypertrophic and inflammatory changes in the scar tissue resulting from the burn and respond poorly to conventional treatments [2]. Poor sleep quality (insomnia) is also a common complaint in patients with PBS syndrome, perhaps due to elevated rates of comorbid posttraumatic stress syndrome and pruritus [2].
Laser therapy and surgical intervention are considered the most effective methods for the treatment of burn scars [3]. However, these interventions must often be delayed until the scar is stabilized, even though early laser and surgery were developed in the most recent decade $[4,5]$. As patients with major burn scars require additional time for their scarring to stabilize, this protracted time preceding treatment is likely to leave them at a greater risk of more severe PBS syndrome symptoms and scar conditions [2-4]. Rehabilitation plays an additional, irreplaceable role in treating burn scar contracture, especially in those with major burn scars. However, PBS syndrome symptoms usually cannot be relieved by rehabilitation or pharmaceutical treatment alone [2]. Comple- 
mentary alternative medicine treatment modalities, such as massage, hypnosis, music therapy, and aromatherapy, have demonstrated some effectiveness in treating PBS syndrome [6]. The application of the meridian system in acupuncture is another complementary practice widely used for pain control that has been successfully utilized in the treatment of pain associated with burn scars $[2,7]$.

In recent years, there has been growing interest in the application of auriculotherapy in both clinical and experimental studies. Although scientific evidence is limited, some studies have affirmed that the use of neurostimulation impacts the release of neurotransmitters, thus affecting the activity of the central nervous system (CNS). According to Shiozawa et al. (2014) [8], electrical stimulation of cranial nerves can be performed by stimulation of the external ear and take advantage of brain plasticity for therapeutic purposes. Mercante et al. (2018) [9] proposed the concept of auricular neuromodulation (AN) by reviewing the neuroanatomical and neurochemical properties of the vagus nerve and trigeminal nerve innervating the auricle and the specific parts of the nervous system regions that interact with the external ear. In addition, multiple scientific studies have shown similar findings between noninvasive electrical stimulation of the auricular region connected with the vagus nerve and invasive electrical stimulation of the actual vagus nerve. Niemtzow developed "battlefield acupuncture" (BFA) in 2007, which was purported to influence central pain control through its effects on a somatotopic representation of the auricle; however, the evidence base is relatively meager [10-12]. In 2017, the Singapore Symposium on auriculotherapy summarized the neurophysiological understanding, and the clinical application of auriculotherapy was correlated with the auricular branch of the vagus nerve. Thus, the auricles could be the most affordable target for noninvasive techniques to manipulate CNS functions. Randomized controlled trials of auriculotherapy for chronic pain, osteoarthritis, poststroke syndrome, and drug abuse have shown statistically significant improvements in these conditions $[13,14]$. In addition, the application of magnetic auriculotherapy has been found to alleviate pain responses and distress in neonates in the intensive care unit [15]. Although acupuncture is now common practice in many parts of the world, it is still not a mainstream practice, partly due to needle phobia. Therefore, ear acupoints treated with laser light, self-administered auricular acupressure (AA), or magnetic beads for clinical applications have been developed as alternative auriculotherapy methods [13].

In the present study, we sought to assess whether PBS syndrome symptoms in major burn victims [with a total burn surface area (TBSA) greater than 20\%] could be treated by the application of auriculotherapy according to the meri- dian system concept as opposed to relying on the use of pharmacological treatments. As the patient population included individuals with major burn scar injuries, we focused on stimulation of auricular acupoints with self-message magnet beads to both facilitate treatment and decrease interference with the burn scars themselves. The auricular Shenmen and Subcortex acupoints were specifically chosen after a review of the literature [14,16]. Both auricular acupoints affect the autonomic nervous system, especially the parasympathetic branch, which may help to relieve PBS syndrome symptoms including pain and pruritus. In the present prospective singlearm interventional study, we sought to assess whether PBS syndrome symptoms could be relieved by auriculotherapy.

\section{MATERIALS AND METHODS}

\section{Patient selection}

All participants screened and analyzed for this study were victims of the Formosa Color Dust Explosion in Taiwan [11]. The inclusion criteria were as follows: 1) age of 19-35 years, 2) TBSA greater than $20 \%$, 3) mature scar stabilization for longer than six months, 4) stable condition with rehabilitation status, 5) no CNS injuries or comorbidities, and 6) no laser treatments or surgical operations in the past three months.

Patients with burn scars covering less than $20 \%$ of their TBSA, alcoholism, neurological defects, any coexisting medical problems prior to their burn injury, or amputation of a major limb due to the burn injury and those who underwent laser treatment or surgery in the previous three months were excluded. We also excluded patients taking psychiatric medications or sleeping pills prior to their burn injury and those who were currently in an unstable medical condition. Finally, patients who were unwilling to complete our questionnaires and/or comply with treatment protocols, those who received prior treatment(s) for PBS syndrome (other than auricular-stimulating therapy), and those who had undergone amputation of a major limb following their burn injury were also excluded from the present study.

Between May 2016 and May 2017, 62 patients underwent study entry. Among them, only 31 met the study inclusion criteria and were enrolled. Of these 31 , one female withdrew on the day following auricular magnetic bead treatment due to intolerable burn scar pain, yielding a final cohort of 30 individuals in the present study.

Approval was applied for and obtained from both study site institutional review boards (IRBs): The Shin Kong Wu Ho-Su Memorial Hospital IRB in Taipei, Taiwan (IRB No. 20160201R) and the Sunshine Social Welfare Foundation IRB (IRB No. SU105001, 10491 3F, No.91, Section 3, Nanjing East Road, Zhongshan District, Taipei City, Taiwan). The same study protocol was adhered to at both study sites. 


\section{Evaluation tool}

The surface of the burn scar was evaluated according to the Lund and Browder chart. Subjective pain was evaluated using a visual analog scale (VAS). The patient marked both the most and the least severe pain they had experienced on a 10-point scale, with 1 indicating the least amount of pain and 10 indicating the most. The Burn Man Itch Scale was used to measure patient itchiness and ranges from "no influence" (0) to "severe influence" (4). Sleep quality was measured using the 5-D Pruritus Scale (Table 1) [2,3,6,7]. To manifest these symptoms more predominantly, neither the Vancouver Scar Scale nor the Observer Scar Assessment Scale was used $[2,3,6,7]$.

No objective evaluation of PBS syndrome or other scar syndromes was conducted. Instead, heart rate variability (HRV) was used to evaluate the influence of auricularstimulating therapy on the autonomic nervous system. The functioning of both the parasympathetic and sympathetic branches of the autonomic nervous system can affect sleepiness, pain threshold, and pruritis. HRV is commonly used to evaluate general autonomic system functioning in psychiatric patients; however, in the present study, we expanded its application to evaluate patients with burn scars. The general status of autonomic nervous system functioning can be quantified as standard deviations from the normal-tonormal heart rate interval (SD), total power of autonomic system activity (TP), and very low heart rate frequency (VL). Normalized low-frequency power (LFN) refers to the sympathetic system, and normalized high-frequency power (HFN) refers to the parasympathetic system.

The whole duration of the study was 29 days, and the intervals included a one-week intervention and a one-week break for two courses. The preinterventional data were collected on the first day, and the postinterventional data were collected separately on the fifteenth and twenty-ninth experimental days.

\section{Treatment protocol}

Prior to treatment, the recruited patients completed clinical variable questionnaires and underwent HRV testing at their first evaluation. Magnetic beads were placed over both the auricular Shenmen and Subcortex acupoints on one ear (Fig. 1) with the aid of an acupoint detection pen. The treatment intervention was self-administered massage five times per day, and both magnetic beads were removed on the eighth experimental day. The patients then received a second evaluation as a postinterventional assessment and entered the second course of treatment. The final data were collected on the twenty-ninth experimental day after the second course of treatment and a one-week break (Fig. 2). In total, each patient received two courses of treatment and had three evaluations.

After completing the study, 16 patients received laser treatments and continued auriculotherapy for up to 12 weeks as an experimental group. In contrast, 14 patients discontinued auriculotherapy. Among them, the seven patients who did not receive any interventions were viewed as the control group (Fig. 3). All patients were evaluated by questionnaires and HRV again after three months to test for a sustained effect.

\section{Theory/Calcullation}

The statistical tests used in the present study were performed with SPSS (22, SPSS Inc., Chicago, IL, USA). Pairedsamples t-tests, $\mathrm{Z}$ tests, and Pearson's correlations were used for analyses.

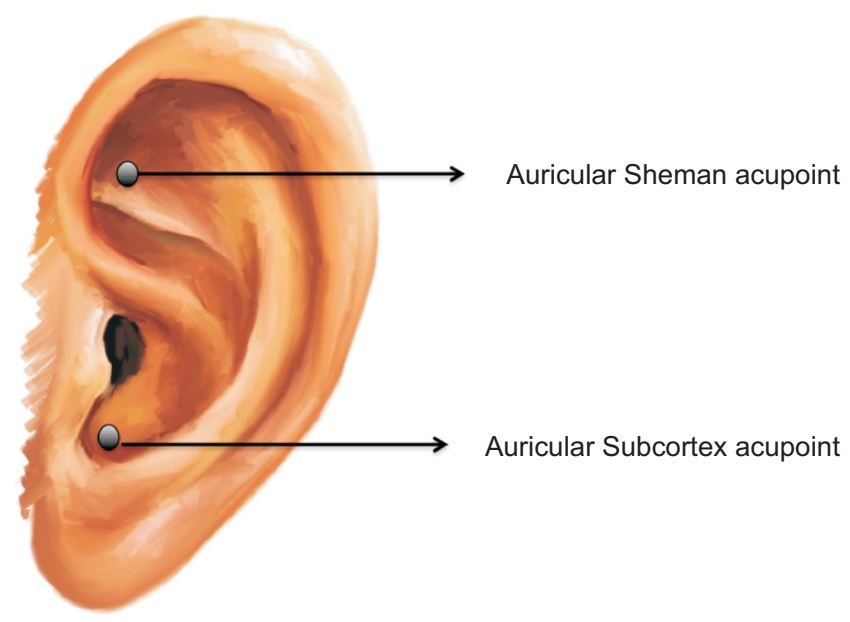

Fig. 1. Auricular acupoints. The auricular Shenmen (HT7) and Subcortex (AT4) acupoints were used to treat PBS syndrome in major burn patients. Stimulation of both acupoints may interfere with vagal tone and thus affect autonomic activity.

Table 1. Sleep according to the 5-D Pruritus Scale

\begin{tabular}{|c|c|c|c|c|c|}
\hline Sleep & 1 & 2 & 3 & 4 & 5 \\
\hline & Never affects sleep & $\begin{array}{l}\text { Occasionally delays } \\
\text { falling sleep }\end{array}$ & $\begin{array}{l}\text { Freqeuntly delays falling } \\
\text { asleep }\end{array}$ & $\begin{array}{l}\text { Delays falling asleep and } \\
\text { occasionally wakes me } \\
\text { up at night }\end{array}$ & $\begin{array}{l}\text { Delays falling asleep and } \\
\text { frequently wakes me up } \\
\text { at night }\end{array}$ \\
\hline
\end{tabular}

The sleep condition in individuals per the 5-D Pruritus Scale reflects their sleep quality. 


\section{RESULTS}

Of the 30 patients, seven were male, and 23 were female (Fig. 3). Their mean age was 23.9 years (range: $19-31$ years), and the mean TBSA was $55.9 \%$ (35-80\%). Seven females finished the first course of the intervention without engagement in any other treatments apart from regular rehabilitation for three

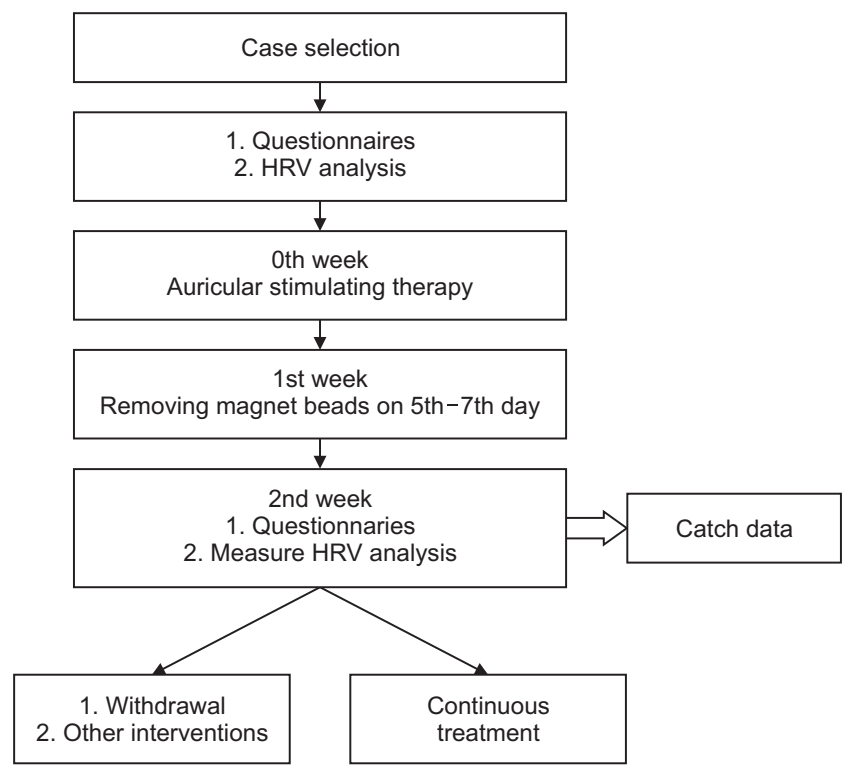

Fig. 2. Study protocol for auriculotherapy. The indicated patients followed the treatment protocols and received evaluations both before and after treatment. Patients who pursued other interventions, such as laser therapy, herbal supplementation, and/or surgery during treatment, were excluded. The data were recorded every two weeks, but only the data in the fourth week were used for the final analysis. months and were used as a control group in the assessments for sustained treatment effects (Fig. 2).

\section{Analyses of background pain, paroxysmal pain, itchiness, and sleep quality}

Table 2 presents the preinterventional and postinterventional evaluation results including the lower pain limit (background pain), upper pain limit (paroxysmal pain), itchiness, and sleep quality. Six patients (20\%) felt improvement in their background pain levels following treatment, whereas 24 patients (80\%) felt no change (data not shown). Thus, following treatment, the average patient background pain decreased significantly from 1.83 to $1.57(p=0.023)$. In terms of paroxysmal pain, 19 patients improved (63.3\%) following treatment, whereas 11 patients experienced no change (26.7\%), with the average paroxysmal score decreasing significantly from 5.00 to $3.31(p<0.001)$ following treatment. The average reported itchiness decreased significantly from 3.57 to 2.4 ( $p$ $<0.001$ ) following treatment; relief was experienced reported among 25 patients (83.3\%), and the remaining five did not notice any change (16.7\%). The average patient sleep disability score decreased from 4.57 to 3.43 ( $p<0.001$ ), with 24 patients $(80 \%)$ reporting an improvement in sleep quality and six $(20 \%)$ reporting no change.

\section{Control group}

The mean age of the patients in the control group was 25.1 years (range: $23-28$ years), and their mean TBSA was 55.36\% (range: 38-67.5\%). All patients were women (data not shown). Within three months of treatment cessation, their background pain, paroxysmal pain, itchiness, and sleep disability scores all returned to their respective pretreatment baselines.

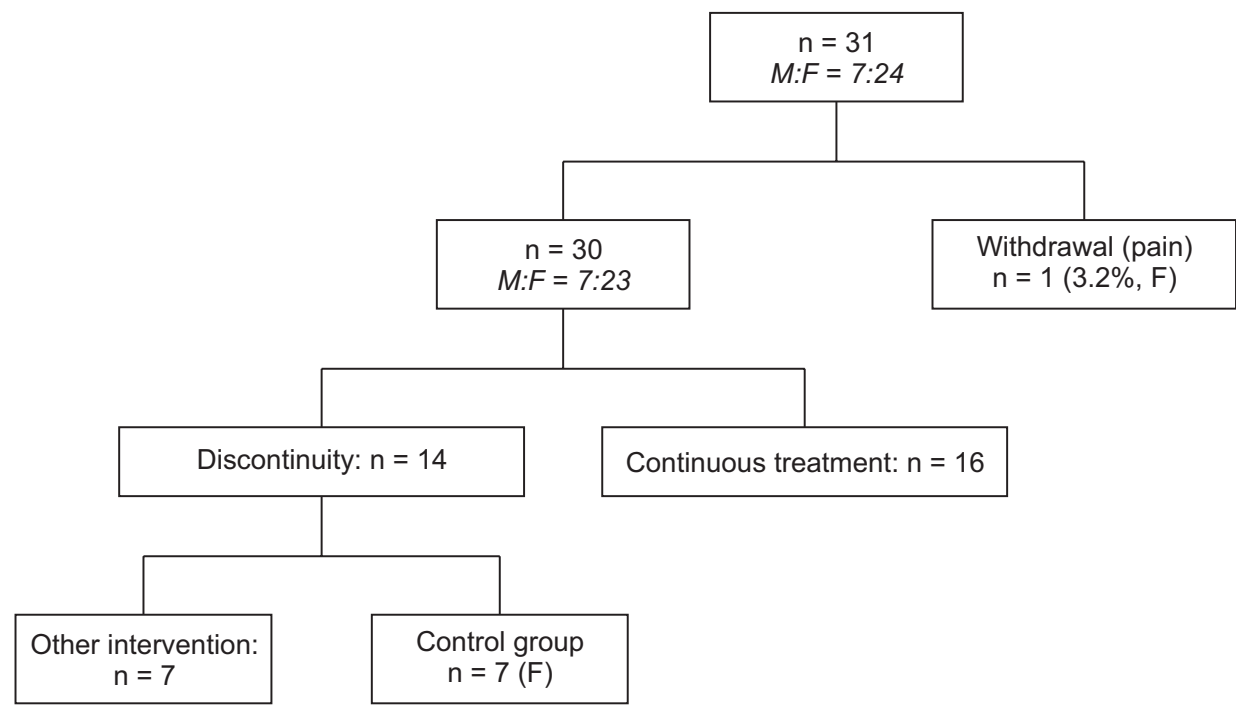

Fig. 3. Analyses of patients. Thirtyone patients were originally included, and one withdrew due to intolerable pain. Seven patients who did not receive other interventions for the three months following acupoint stimulation were analyzed as the control group. $\mathrm{n}=$ number; $\mathrm{M}=$ male; $F=$ female. 


\section{HRV analysis}

Table 3 presents the results of the HRV analysis. Following the acupuncture intervention, the average SD increased from 39.3 to 42.9 , TP increased from 1534.53 to 1691.60 , VL

Table 2. Analyses of the interventional effectiveness on PBS syndrome symptoms

\begin{tabular}{lcccr}
\hline & Pre-test & Post-test & Change & $p^{\mathrm{a}}$ \\
\hline VASL & $1.83(\mathrm{SD}=0.915)$ & $1.57(\mathrm{SD}=0.682)$ & -0.26 & $0.023^{*}$ \\
VASH & $5.00(\mathrm{SD}=2.093)$ & $3.13(\mathrm{SD}=1.346)$ & -1.87 & $<0.001^{*}$ \\
$\mathrm{SI}$ & $3.57(\mathrm{SD}=0.561)$ & $2.43(\mathrm{SD}=0.572)$ & -1.14 & $<0.001^{*}$ \\
S & $4.57(\mathrm{SD}=0.63)$ & $3.43(\mathrm{SD}=0.75)$ & -1.14 & $<0.001^{*}$
\end{tabular}

Auriculotherapy can improve PBS syndrome symptoms such as procedure-related pain, background pain, itchiness, and sleep quality $(p<0.05$ in all groups). One course of auriculotherapy alone can help relieve from severe PBS syndrome.

(Total study group, $\mathrm{n}=30$ ).

${ }^{*} p<0.05$, significant.

$\mathrm{a}=$ Paired-samples t-test; $\mathrm{VASL}=$ Visual analog scale lower limit or background pain; VASH = Visual analog scale upper limit or paroxysmal pain; $\mathrm{SI}=$ Itchiness as measured by the Burn Man Itch Scale; $S=$ Sleep disability score as measured by the $5-D$ Pruritis Scale. increased from 662.13 to 719.30 , LFN increased from 48.63 to 49.5 , and HF increased from 46.57 to 42.23 . However, these changes failed to reach statistical significance. Following patient stratification by HRV status (normal HRV SD $\geq$ 40 and abnormal HRV SD < 40), differences in the SD, TP, and VL did reach statistical significance $(p<0.05)$ in the abnormal group.

Comparing the pre- and postintervention clinical symptoms, pain, itching, and sleeping quality were improved with an increased SD, but the Pearson correlation coefficient showed no correlation (-0.004). In the preintervention group, the LFN and HF were weakly correlated with scar itching $(-0.192)$ and sleeping quality (0.147). Although the changes in clinical symptoms and HRV between the two groups were statistically significant, the correlation between the curative effect of auriculotherapy and postintervention restoration of the autonomic nervous system was unclear (Table 4).

\section{DISCUSSION}

During the follow-up period, auricular magnetic beads were found to have no sustained effects on symptom relief

Table 3. Changes in the autonomic systems after auriculotherapy

\begin{tabular}{|c|c|c|c|c|}
\hline & $\begin{array}{l}\text { Mean } \\
\text { Change }\end{array}$ & Std. deviation & $95 \%$ confidence interval & $p$ \\
\hline \multicolumn{5}{|c|}{ Total study group $(\mathrm{n}=30)$} \\
\hline SD & -3.9 & 17.462 & -10.407 to 2.607 & 0.23 \\
\hline TP & -157.067 & 1409.238 & -683.285 to 369.152 & 0.546 \\
\hline VL & -57.167 & 610.763 & -285.229 to 170.896 & 0.612 \\
\hline $\mathrm{LH}$ & 0.07 & 1.303 & -0.416 to 0.557 & 0.771 \\
\hline LFN & -0.867 & 18.604 & -7.814 to 6.08 & 0.8 \\
\hline HFN & 4.333 & 46.846 & -13.159 to 21.826 & 0.616 \\
\hline \multicolumn{5}{|c|}{ Normal HRV SD $\geq 40(n=10)$} \\
\hline SD & 6.100 & 24.081 & -11.126 to 23.326 & 0.444 \\
\hline $\mathrm{TP}$ & 783.500 & 1943.763 & -606.984 to 2173.984 & 0.234 \\
\hline VL & 266.70 & 829.620 & -326.775 to 860.175 & 0.336 \\
\hline LH & 0.180 & 0.805 & -0.396 to 0.756 & 0.498 \\
\hline LFN & 3.500 & 13.041 & -5.829 to 12.829 & 0.418 \\
\hline HFN & 12.300 & 75.648 & -41.816 to 66.416 & 0.620 \\
\hline \multicolumn{5}{|c|}{ Abnormal HRV $(S D<40)(n=20)$} \\
\hline SD & -8.900 & 10.483 & -13.806 to -3.994 & $0.001^{*}$ \\
\hline TP & -672.350 & 736.946 & -972.251 to -282.449 & $0.001^{*}$ \\
\hline $\mathrm{VL}$ & -219.100 & 400.667 & -406.618 to -31.582 & $0.024^{*}$ \\
\hline $\mathrm{LH}$ & 0.015 & 1.509 & -0.69 to 0.721 & 0.965 \\
\hline LFN & -3.050 & 20.801 & -12.785 to 6.685 & 0.520 \\
\hline HFN & 0.350 & 24.265 & -11.006 to 11.706 & 0.929 \\
\hline
\end{tabular}

The SD indicates the total condition of the autonomic nervous system. Auriculotherapy showed a tendency of restoration of the autonomic nervous systems according to the SD data; however, the final data did not show significant differences. Further analysis by age showed that patients with an abnormal autonomic nervous systems with an $\mathrm{SD}<40$ may have more significant benefit from auriculotherapy ( $p<0.05$ ). Those with a normal SD level before auriculotherapy showed no significant change in the SD after auriculotherapy.

${ }^{*} p<0.05$, significant.

$\mathrm{HRV}=$ Heart rate variability; $\mathrm{SD}=$ Standard deviation of the normal-to-normal heart rate interval; $\mathrm{TP}=$ Total power of the autonomic nervous system activity; $\mathrm{VL}=$ Very low frequency of heart rate analysis; LFN = Normalized low-frequency power referring to the sympathetic nervous system; HFN = Normalized high-frequency power referring to the parasympathetic nervous system. 
in patients with major burns after discontinuous use. This is partly because auriculotherapy cannot change the topical burn scar; this modality only relieves the symptoms caused. The nature and symptoms of scars are assumed to keep changing for up to 10 years. Other considerations are the intervention dosage and duration not being sufficient to exploit neuroplasticity. However, the symptoms of the other 23 patients who received laser therapy and auriculotherapy were relieved with decreased medication adherence. The mainstream methods to managed PBS syndrome only include laser therapy, surgery, and time, but auriculotherapy could offer an affordable way to alleviate symptoms and help patients adapt to the change (Fig. 4).

Scar tissue originating from major burn injuries may induce hypertrophy and cause inflammation, which consequently produce pain and pruritus that do not respond well to conventional treatments [2]. While there is no clear mechanism for this phenomenon, evidence indicates that a specific set of $\mathrm{C}$ group fibers in the dermis and epidermis transmit pain and itch via complex molecular activities. Pain and itch can interfere with sleep, thereby causing insomnia, which is further exacerbated by posttraumatic stress disorder and depression symptoms that are common in patients with burn injuries [17]. In addition to standard pharmaceutical treatments, many patients seek relief with alternative therapies, such as aromatherapy and acupuncture, forms of traditional complementary medicine (TCM) that are increasingly used for similar indications for which medical options are limited $[18,19]$.

Despite insufficient evidence on the mechanistic underpinnings of auricular point acupressure [20], many studies have demonstrated its effectiveness for the treatment of sleep disturbances as well as acute and chronic pain [21-24]. A study with systematic reviews showed that auriculotherapy may be effective for treating a variety of types of pain including acute and chronic pain via different combinations of acupoints and meridian systems [23]. For example, auricular stimulation at the Shenmen and "point zero" acupoints has been shown to induce parasympathetic activation and pain relief,
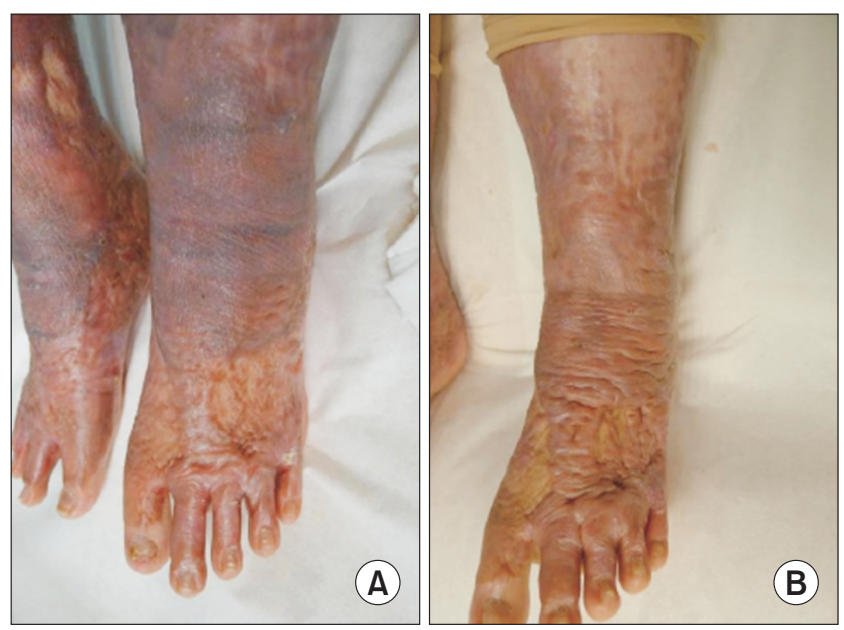

Fig. 4. Burn scar appearance. Prior to the study, the appearance of burn scars in each patient showed erythematous changes (rich vascularity), easy maceration, and contracture (A). After completing the study, the burn scars of patients who received no intervention were relatively stable but still contracted (B). Auriculotherapy can relieve systemic symptoms but was not associated with sustained effects due to local contracture. At this stage, laser interventions and surgeries are advised to control scar syndrome.

Table 4. Correlation between HRV analysis values and PBS syndrome symptoms. The SD, TP, VL, LFN, and HF can be used to assess the sympathetic and parasympathetic nervous systems

\begin{tabular}{lcccccccrrrr}
\hline & pre-SD & Post-SD & Pre-TP & Pre-VL & Pre-LFN & Pre-HF & Pre-VASL & Pre-VASH & Pre-SI & Pre-S \\
\hline pre-SD & 1 & 0.473 & 0.971 & 0.871 & -0.100 & 0.474 & 0.210 & 0.117 & 0.062 & 0.062 \\
post-SD & 0.473 & 1 & 0.429 & $0.560^{*}$ & 0.051 & -0.082 & 0.136 & 0.169 & -0.004 & -0.004 \\
pre-TP & 0.971 & 0.429 & 1 & 0.890 & -0.053 & $0.51^{*}$ & 0.182 & 0.080 & 0.056 & 0.056 \\
pre-VL & 0.871 & 0.560 & 0.890 & 1 & 0.017 & 0.358 & 0.119 & -0.023 & 0.011 & 0.011 \\
pre-LFN & -0.100 & 0.051 & -0.053 & 0.017 & 1 & -0.225 & -0.247 & -0.097 & -0.192 & -0.192 \\
pre-HF & 0.474 & -0.082 & 0.513 & 0.358 & -0.225 & 1 & 0.008 & 0.166 & 0.147 & 0.147 \\
pre-VASL & 0.210 & 0.136 & 0.182 & 0.119 & -0.247 & 0.008 & 1 & 0.653 & 0.111 & 0.111 \\
pre-VASH & 0.117 & 0.169 & 0.080 & -0.023 & -0.097 & 0.166 & 0.653 & 1 & 0.165 & 0.165 \\
pre-SI & 0.062 & -0.004 & 0.056 & 0.011 & -0.192 & 0.147 & 0.111 & 0.165 & 1 \\
pre-S & 0.062 & -0.004 & 0.056 & 0.011 & -0.192 & 0.147 & 0.111 & 0.165 & 1.000 & 1.000 \\
\hline
\end{tabular}

Short-term auriculotherapy can relieve clinical symptoms but compared with HRV data, only low correlations were detected.

${ }^{*} p<0.05$, significant.

PBS = post-burn scar; SD = Standard deviation of the normal-to-normal interval of the heart rate; TP = Total power of autonomic nervous system activity; $\mathrm{VL}=$ Very low frequency of heart rate analysis; LFN = Normalized low-frequency power referring to the sympathetic nervous system; HFN = Normalized high-frequency power referring to the parasympathetic nervous system; VASL = Visual analog scale lower limit or background pain; $\mathrm{VASH}=$ Visual analog scale upper limit or paroxysmal pain; $\mathrm{SI}=$ Itchiness as measured by the Burn Man Itch Scale; $\mathrm{S}=$ Sleep disability score as measured by the 5-D Pruritis Scale. 
whereas local anesthesia directly injected over the auricular Shenmen point has been demonstrated to relieve acute pain intraoperatively [25]. In addition, the effects of insomnia, depression, and pruritus have been widely applied [23-26]. Lan et al. [27] reviewed 15 studies and concluded by suggesting the benefits of auriculotherapy for primary insomnia. Yan et al. [26] showed the effectiveness of auriculotherapy for uremic pruritus in patients on hemodialysis. Some reviewers have hypothesized that auricular stimulation on certain acupoints may treat and relieve pain, insomnia, and pruritus via autonomic pathways [23-28]. Using these studies as a theoretical basis, we hypothesized that auricular stimulation of the Shenmen point might be helpful in providing relief of parasympathetically regulated PBS syndrome symptoms, such as pain, itch, and insomnia.

Among women with postmenopausal insomnia (PI), auriculotherapy innervation has been shown to improve PI with more cardiac parasympathetic activity and less cardiac sympathetic activity [29]. In our studies, all patients had major burn scars and lost at least $20 \%$ of their perspiration systems due to damage to the sweat glands from burns, and impaired temperature perception was also found. These physical impairments are significant contributors to burn-related insomnia. Parkinson's disease (PD) and Alzheimer's disease $\mathrm{AD})$ are the most frequent age-related neurodegenerative disorders and are characterized by the frequent occurrence of sleep problems. Similar clinical symptoms between patients with PD and burn patients are impaired temperature perception and altered sleep regulation [30-32].

According to meridian therapy, the acupoint Shenmen located at the fossa of the ear is the first acupoint in the ear, standing for the "heart" of the ear and dominating various treatments. This point can be stimulated to control pain as well as other symptoms such as convulsions, sedation, anxiety, depression, worry, sleeplessness, impatience, stick, addiction withdrawal, restlessness, dysphoria, hysteria, neuralgia, and headache [28]. Studies have shown that auriculotherapy is a fitting choice because this treatment increases parasympathetic nerve activity, thereby modulating the autonomic nervous system and achieving a synergistic effect $[24,27,28]$. In addition to the auricular Shenmen point, treatment of insomnia via stimulation of other auricular acupoints, such as Heart, Occiput, Kidney, Brain, and Subcortex points, is also common $[19,23,24]$. The acupoint Subcortex is part of the thalamic points (Subcortex, Brain, and Pain control points) at the junction between the helix and antitragus of the ear $[33,34]$. The ear is innervated by CN VII, CN V, auricular branches of the vagus nerve (ABVNs), sensory area of the facial nerve, glossopharyngeal nerve, lesser occipital nerve, and greater auricular nerve. The ABVNs mainly affect the concha and most of the auditory canal. Stimulation of the ABVNs will put out the reticular formation, parasympathetic preganglionic neurons to the viscera, paraventricular nucleus of the hypothalamus, thalamus, and the amygdala. Stimulation may also produce anti-inflammatory effects to alleviate pain [34]. As a support point, it can reduce neurasthenia, anxiety, depression, schizophrenia, and pain by affecting thalamic relay, which is a supraspinal gate control system that affects the cerebral cortex in terms of pain $[33,34]$.

To facilitate treatment of patients with scarring at the ears, the Shenmen and Subcortex acupoints were chosen for PBS syndrome. Connections between the auricles and the autonomic nervous system have been the subject of significant research interest, beginning with the identification of Arnold's Reflex in 1832 and continuing into present times aided by the use of functional magnetic resonance imaging (fMRI). Evidence suggests that stimulation of auricular acupoints increases vagal tone and regulates systematic functioning in the human body, increasing pain thresholds and decreasing inflammation and anxiety. Since Lewis et al. [16] first published the application of auricularstimulating therapy in the treatment of burn patients, the use of this technique has expanded; it is now applied as a first-aid technique for burn victims in several countries [34]. However, despite the wide adoption of auricular therapy, no assessments of PBS syndrome treatment in major burn victims have been published. This may be because patients with major burn scars, much like those with chronic cancer pain, often seek multimodal treatments that include both formal and informal therapies. These multimodal approaches render evaluating the effects of a singular therapy in these clinical populations impossible. Further exacerbating this complexity, most patients with major burn scar syndromes also experience other comorbidities. Thus, the investigation undertaken in this research offers a rare chance to study the effectiveness of acupuncture in a setting where confounders are controlled. In addition to treatment control, this study offers the additional benefit of including patients without additional comorbidities. The Formosa Fun Coast Water Park dust explosion, which occurred on June 27, 2015, caused 499 individuals to experience major burns. As the event took place at a recreational water park, the vast majority of these patients were young, healthy adults with few comorbidities [17]. Given this, we had the rare opportunity to investigate the effectiveness of a one-course treatment of auriculotherapy in relieving pain and itchiness from PBS syndrome among a largely unconfounded population. While we were successful in demonstrating the effectiveness of auriculotherapy, we also found that patient symptoms returned to their preintervention baseline three months after treatment cessation, suggesting the need for ongoing treatment in these 
individuals.

Although we did not find prominent correlations, this study also made novel use of HRV to assess autonomic changes among major burn scar patients undergoing auriculotherapy. HRV is a noninvasive technique used to quantify fluctuations in the autonomic nervous system and a potential tool for assessing treatment effects instead of fMRI [35-40]. Karri et al. [36] and Gouin et al. [37] applied HRV to evaluate treatment effectiveness in neuropathic pain and sleep disturbances. Lin et al. [39] showed increased autonomic activation in the field of biofeedback therapy for major depression and insomnia. HRV has been widely used to interpret these subjective symptoms as objective data $[36,37,39,40]$. In the latest studies, Loguidice et al. [38] used HRV as a predictor of death in burn patients, and Joo et al. [40] used 24-h HRV in patients with burns to evaluate various symptoms in the daytime and nighttime [39]. HRV studies have shown that PBS syndrome is correlated with treatment. Recently, HRV data have been used to compare the effectiveness of pre- and posttreatments in an increasing number of clinical studies, and these subjective symptoms were interpreted as objective data. As burn scar assessment is the main subject, additional studies are needed to develop a more objective method for burn scar assessment.

A major advantage of this study is that every patient included was in the same treatment group. With this in mind, we did not have to account for confounders derived from the use of other treatment modalities. However, the strength of our findings is also somewhat limited by our study design. As our investigation was not double-blinded, it is possible that our patients were influenced by demand characteristics and the placebo effect. Despite the possibility of bias, we observed auriculotherapy to be correlated with improvements in several clinical parameters. It is the wish of the authors that auriculotherapy be considered as an adjuvant therapy in patients with PBS syndrome.

\section{CONCLUSIONS}

Even a single course of auriculotherapy is effective in relieving PBS syndrome symptoms among patients with major burn scars. We did not detect a sustained effect three months after cessation of treatment. However, continued auriculotherapy may be a useful adjuvant among patients with PBS syndrome who often have intractable symptoms that cannot be relieved with pharmaceutical therapy and who must wait for their scars to stabilize before laser treatments and surgical operations become feasible. A tendency of recovery of the autonomic system after treatment was not confirmed via HRV detection, and the correlations among clinical symptoms and HRV data were low. More studies are needed for further evaluation in the future.

\section{ACKNOWLEDGEMENTS}

We thank both Shin Kong Wu Ho-Su Memorial Hospital (research grant No. 20160201R) and the Yi Yen-Liang foundation development and construction plan of the School of Medicine at National Yang-Ming University for supporting the study in its entirety.

\section{CONFLICT OF INTEREST}

The authors declare no conflict of interest.

\section{ORCID}

Cha-Chun Chen, https://orcid.org/0000-0002-6886-6690 San-Pei Chen, https://orcid.org/0000-0001-5468-1110 Shu-Yu Lyu, https://orcid.org/0000-0002-1360-6736 Chung-Hua Hsu, https://orcid.org/0000-0002-2496-7246

\section{REFERENCES}

1. Choi YH, Kim KM, Kim HO, Jang YC, Kwak IS. Clinical and histological correlation in post-burn hypertrophic scar for pain and itching sensation. Ann Dermatol 2013;25:428-33.

2. Cuignet O, Pirlot A, Ortiz S, Rose T. The effects of electroacupuncture on analgesia and peripheral sensory thresholds in patients with burn scar pain. Burns 2015;41:1298-305.

3. Willows BM, Ilyas M, Sharma A. Laser in the management of burn scars. Burns 2017;43:1379-89.

4. Lim Y, Park JH, Lee DY, Hwang NY, Ahn S, Lee JH. The important factors associated with treatment response in laser treatment of facial scars: a single-institution based retrospective study. Ann Dermatol 2019;31:6-13.

5. Gold MH, McGuire M, Mustoe TA, Pusic A, Sachdev M, Waibel $\mathrm{J}$, et al. Updated international clinical recommendations on scar management: part 2--algorithms for scar prevention and treatment. Dermatol Surg 2014;40:825-31.

6. Stoddard FJ Jr, Ryan CM, Schneider JC. Physical and psychiatric recovery from burns. Surg Clin North Am 2014;94:863-78.

7. Fang S. The successful treatment of pain associated with scar tissue using acupuncture. J Acupunct Meridian Stud 2014;7:262-4.

8. Shiozawa P, Silva ME, Carvalho TC, Cordeiro Q, Brunoni AR, Fregni F. Transcutaneous vagus and trigeminal nerve stimulation for neuropsychiatric disorders: a systematic review. Arq Neuropsiquiatr 2014;72:542-7.

9. Mercante B, Deriu F, Rangon CM. Auricular neuromodulation: the emerging concept beyond the stimulation of vagus and trigeminal nerves. Medicines (Basel) 2018;5:10.

10. Niemtzow RC. Battlefield acupuncture. Med Acupunct 2007;19: 
225-8.

11. Walker PH, Pock A, Ling CG, Kwon KN, Vaughan M. Battlefield acupuncture: opening the door for acupuncture in Department of Defense/Veteran's Administration health care. Nurs Outlook 2016;64:491-8.

12. Yeh CH, Chiang YC, Hoffman SL, Liang Z, Klem ML, Tam WW, et al. Efficacy of auricular therapy for pain management: a systematic review and meta-analysis. Evid Based Complement Alternat Med 2014;2014:934670.

13. Oleson T. Progress in clinical applications of auricular acupuncture at the International Symposium on Auriculotherapy held in Singapore. SOJ Anesthesiol Pain Manag 2018;5:1-4.

14. Chang LH, Hsu CH, Jong GP, Ho S, Tsay SL, Lin KC. Auricular acupressure for managing postoperative pain and knee motion in patients with total knee replacement: a randomized sham control study. Evid Based Complement Alternat Med 2012;2012: 528452.

15. Gan KML, Oei JL, Quah-Smith I, Kamar AA, Lordudass AAD, Liem KD, et al. Magnetic non-invasive auricular acupuncture during eye-exam for retinopathy of prematurity in preterm infants: a multicentre randomized controlled trial. Front Pediatr 2020;8:615008.

16. Lewis SM, Clelland JA, Knowles CJ, Jackson JR, Dimick AR. Effects of auricular acupuncture-like transcutaneous electric nerve stimulation on pain levels following wound care in patients with burns: a pilot study. J Burn Care Rehabil 1990;11: 322-9.

17. Aili Low JF, Meyer WJ, Willebrand M, Thomas CR. Psychiatric disorders associated with burn injury. In: Herndon DN, ed. Total Burn Care, 5th ed. Amsterdam: Elsevier, 2018:700-8.e3.

18. Stanton G. Auriculotherapy in neurology as an evidence-based medicine: a brief overview. Med Acupunct 2018;30:130-2.

19. Liugan M, Zhang M, Cakmak YO. Neuroprosthetics for auricular muscles: neural networks and clinical aspects. Front Neurol 2018;8:752.

20. Paley CA, Johnson MI, Tashani OA, Bagnall AM. Acupuncture for cancer pain in adults. Cochrane Database Syst Rev 2015; 2015:CD007753.

21. King HC, Spence DL, Hickey AH, Sargent P, Elesh R, Connelly CD. Auricular acupuncture for sleep disturbance in veterans with post-traumatic stress disorder: a feasibility study. Mil Med 2015;180:582-90.

22. Yeh CH, Chien LC, Chiang YC, Ren D, Suen LK. Auricular point acupressure as an adjunct analgesic treatment for cancer patients: a feasibility study. Pain Manag Nurs 2015;16:285-93.

23. Asher GN, Jonas DE, Coeytaux RR, Reilly AC, Loh YL, Motsinger-Reif AA, et al. Auriculotherapy for pain management: a systematic review and meta-analysis of randomized controlled trials. J Altern Complement Med 2010;16:1097-108.

24. Vieira A, Reis AM, Matos LC, Machado J, Moreira A. Does auriculotherapy have therapeutic effectiveness? An overview of systematic reviews. Complement Ther Clin Pract 2018;33:61-70. 25. Arai YC, Sakakima Y, Kawanishi J, Nishihara M, Ito A, Tawada $\mathrm{Y}$, et al. Auricular acupuncture at the "shenmen" and "point zero" points induced parasympathetic activation. Evid Based Complement Alternat Med 2013;2013:945063.

26. Yan CN, Yao WG, Bao YJ, Shi XJ, Yu H, Yin PH, et al. Effect of auricular acupressure on uremic pruritus in patients receiving hemodialysis treatment: a randomized controlled trial. Evid Based Complement Alternat Med 2015;2015:593196.

27. Lan Y, Wu X, Tan HJ, Wu N, Xing JJ, Wu FS, et al. Auricular acupuncture with seed or pellet attachments for primary insomnia: a systematic review and meta-analysis. BMC Complement Altern Med 2015;15:103.

28. Swartz MH. Textbook of Physical Diagnosis: History and Examination, 7th ed. Philadelphia: Elsevier, 2014.

29. Kung YY, Yang CC, Chiu JH, Kuo TB. The relationship of subjective sleep quality and cardiac autonomic nervous system in postmenopausal women with insomnia under auricular acupressure. Menopause 2011;18:638-45.

30. La Morgia C, Ross-Cisneros FN, Sadun AA, Carelli V. Retinal ganglion cells and circadian rhythms in Alzheimer's disease, Parkinson's disease, and beyond. Front Neurol 2017;8:162.

31. Romeijn N, Borgers AJ, Fliers E, Alkemade A, Bisschop PH, Van Someren EJ. Medical history of optic chiasm compression in patients with pituitary insufficiency affects skin temperature and its relation to sleep. Chronobiol Int 2012;29:1098-108.

32. Maldonado MD, Murillo-Cabezas F, Calvo JR, Lardone PJ, Tan DX, Guerrero JM, et al. Melatonin as pharmacologic support in burn patients: a proposed solution to thermal injury-related lymphocytopenia and oxidative damage. Crit Care Med 2007; 35:1177-85.

33. Oleson T. Auriculotherapy Manual: Chinese and Western Systems of Ear Acupuncture, 4th ed. Edinburgh: Churchill Livingstone, 2013.

34. Hou PW, Hsu HC, Lin YW, Tang NY, Cheng CY, Hsieh CL. The history, mechanism, and clinical application of auricular therapy in traditional Chinese medicine. Evid Based Complement Alternat Med 2015;2015:495684.

35. Loskotova A, Loskotova J. The use of acupuncture in first aid of burns-clinical report. Burns 2017;43:1782-91.

36. Karri J, Zhang L, Li S, Chen YT, Stampas A, Li S. Heart rate variability: a novel modality for diagnosing neuropathic pain after spinal cord injury. Front Physiol 2017;8:495.

37. Gouin JP, Wenzel K, Boucetta S, O'Byrne J, Salimi A, Dang-Vu TT. High-frequency heart rate variability during worry predicts stress-related increases in sleep disturbances. Sleep Med 2015;16: 659-64.

38. Loguidice MJ, Schutt RC, Horton JW, Minei JP, Keeley EC. Heart rate variability as a predictor of death in burn patients. J Burn Care Res 2016;37:e227-33.

39. Lin IM, Fan SY, Yen CF, Yeh YC, Tang TC, Huang MF, et al. 
Heart rate variability biofeedback increased autonomic activation and improved symptoms of depression and insomnia among patients with major depression disorder. Clin Psychopharmacol Neurosci 2019;17:222-32.
40. Joo SY, Hong AR, Lee BC, Choi JH, Seo CH. Autonomic nerve activity indexed using 24 -h heart rate variability in patients with burns. Burns 2018;44:834-40. 